\title{
Phase transformation pathway in Ti-15Mo studied by in situ synchrotron $\mathrm{x}$-ray diffraction
}

\author{
P. Zháňal, J. Šmilauerová, P. Harcuba, L. Horák, V. Holý \\ Faculty of Mathematics and Physics, Charles University, Ke Karlovu 5, Prague, Czech Republic \\ jana.smilauerova@karlov.mff.cuni.cz
}

Phase transformations in a single crystal of a metastable $\beta$ titanium alloy (Ti-15Mo in wt \%) were investigated in situ during heating by synchrotron $\mathrm{x}$-ray diffraction. Metastable $\beta$ titanium alloys contain such type and amount of alloying elements that the high-temperature $\beta$ phase (body-centred cubic) can be retained in a metastable state during fast cooling to room temperature; i.e. the formation of low-temperature $\alpha$ phase (hexagonal close-packed) is prevented. Ti alloys from this class generally undergo a wide range of phase transformations due to their metastable nature. First, nano-sized particles of metastable $\omega$ phase form in this class of Ti alloys during fast cooling by a difusionless displacement mechanism, which can be characterized as a collapse of neighbouring (111) $\beta$ planes into their intermediate position. During ageing or heating, $\omega$ particles grow by a combined displacement and diffusion process which is accompanied by rejection of alloying elements from the $\omega$ phase into the surrounding $\beta$ matrix. At higher temperatures, lamellae of the thermodynamically stable $\alpha$ phase precipitate in the material; this process can be assisted either directly or indirectly by the previous $\beta+\omega$ microstructure.

In situ x-ray diffraction was measured using $60 \mathrm{keV}$ photons at the high-energy beamline ID11, ESRF, Grenoble, France. This experiment was performed using an oriented single crystal of Ti-15Mo prepared in an optical floating zone furnace. A slice of the single-crystalline material with the $[100]_{\beta}$ crystallographic axis parallel to the primary beam was placed in a special quartz chamber furnace which allowed measuring in a high vacuum. X-ray diffraction patterns were acquired in situ during heating with a constant heating rate of $5{ }^{\circ} \mathrm{C} / \mathrm{min}$. An example of a measured diffraction pattern is shown in Fig. 1(a).
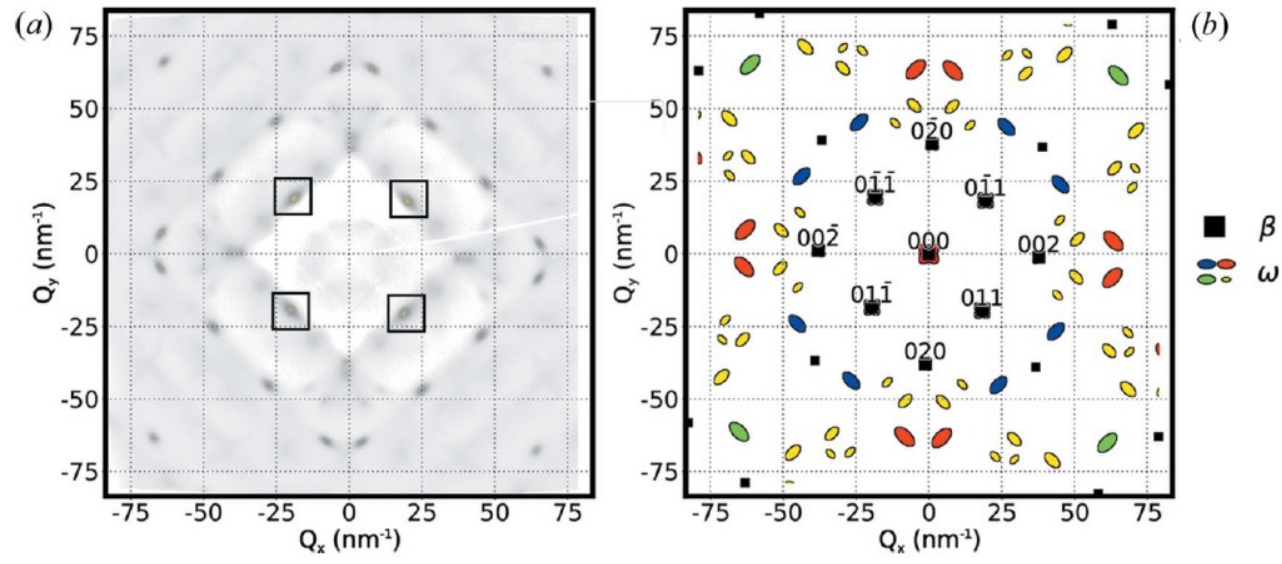

Figure 1. (a) Measured diffraction pattern at room temperature and (b) corresponding calculated pattern with $\beta$ and $\omega$ diffraction maxima denoted by black squares and coloured ellipses, respectively. The four colours represent the four possible orientations of the $\omega$ phase with respect to the matrix. The shapes represent the actual shape of $\omega$ diffraction spots arising from the shape of $\omega$ particles.

Fitting of the temperature dependence of intensity of selected representative single-crystalline diffraction spots showed that at the beginning of linear heating, up to approximately $350^{\circ} \mathrm{C}$, the volume of $\omega$ phase decreased, which is likely connected with displacement-accompanied $\omega$ to $\beta$ reversion. Between $350^{\circ} \mathrm{C}$ and $420^{\circ} \mathrm{C}$, the volume fraction of $\omega$ particles increased which is the consequence of diffusion-driven coarsening of $\omega$ phase particles. Subsequently, as the temperature approached the stability limit of the $\omega$ phase, the volume of $\omega$ decreased. A complete dissolution was observed at $560^{\circ} \mathrm{C}$. Finally, a rapid growth of the $\alpha$ phase commenced at about $580^{\circ} \mathrm{C}$. It was also verified that during linear heating, none of the crystallographic variants of $\omega$ and $\alpha$ phase is preferred.

Keywords: Ti alloys; phase transformations; synchrotron x-ray diffraction

The financial support by the Ministry of Education, Youth and Sports of the Czech Republic (project No. LTAUSA18045) and by ERDF (project 'NanoCent' No. CZ.02.1.01/0.0/0.0/15 003/0000485) is gratefully acknowledged. 